\title{
Differential and Difference Equations: A Themed Issue Dedicated to Prof. Hari M. Srivastava on the Occasion of His 80th Birthday
}

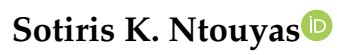 \\ Department of Mathematics, University of Ioannina, 45110 Ioannina, Greece; sntouyas@uoi.gr
}

Received: 16 November 2020; Accepted: 17 November 2020; Published: 18 November 2020

Differential and difference equations play an important role in many branches of mathematics. This Special Issue deals with the theory and applications of differential and difference equations and is dedicated to Professor Hari Mohan Srivastava on the occasion of his 80th Birthday, in recognition of his significant contribution in the field.

\section{Special Issue Overview}

The Special Issue contains 23 contributions in different branches of differential equations. Below, we highlight the main results of the papers.

- In [1], the authors construct generating functions for new families of combinatorial numbers and polynomials and use these generating functions with their functional and differential equations to investigate properties of these new families, and derive many new identities, relations, derivative formulas, and combinatorial sums with the inclusion of binomials coefficients, falling factorial, the Stirling numbers, the Bell polynomials (i.e., exponential polynomials), the Poisson-Charlier polynomials, combinatorial numbers and polynomials, the Bersntein basis functions, and the probability distribution functions.

- In [2], by using the Livshits Theorem, the author provides proof of the completeness of the eigenfunctions and associated functions of the operators, generated by the ordinary differential expressions of the fractional order and boundary conditions of Sturm-Liouville type.

- The authors of [3], studied the fractional view of Whitham-Broer-Kaup equations by using the $q$-homotopy analysis transform method and natural decomposition method. With the help of the Laplace and natural transformations, the procedure strengthened and became easy for implementation. A very close contact of the obtained solutions with the exact solution of the problem was observed.

- In [4], the authors showed convolution formulas and linear recurrence relations satisfied by a generating function containing several parameters. This can be used for number sequences or polynomial sequences, depending on several parameters.

- The authors in [5], established the convergent and stable solutions for the generalized Abel's integral equations of the second kind with variable coefficients, by using Babenko's approach, in the spaces of Lebesgue integrable functions.

- In [6], the authors define classes of harmonic star-like functions with respect to symmetric points and obtain some analytic conditions for these classes of functions. Some results connected to subordination properties, coefficient estimates, integral representation, and distortion theorems are also obtained.

- The asymptotic properties of the class of higher order differential equations with a $p$-Laplacian-like operator are studied in [7], by using the Riccati transformations, the integral averaging technique and comparison principles. 
- In [8], it is shown how the dynamics of the very popular I2SR rumor spreading model can be investigated under stochastic perturbations. It is proved that, for some equilibria of the considered model, it is possible to get conditions for stability in probability in an analytical form, while for other equilibria, the stability condition can be obtained numerically by an appropriate linear matrix inequality via MATLAB.

- A mathematical model of HIV-TB, considering the HIV-infected population is studied in [9]. This model considers HIV-infected individuals as the initial stage. Four equilibrium points are found. Reproduction number $R_{0}$ is calculated. If $R_{0}>1$, disease persists uniformly. With reference to the reproduction number, backward bifurcation is computed for pre-AIDS (latent) stage. Global stability is established for the equilibrium points where there is no pre-AIDS TB class, point without co-infection and for the endemic point.

- In [10], a subclass of complex-valued harmonic univalent functions defined by a generalized linear operator is introduced. Some interesting results, such as coefficient bounds, compactness, and other properties of this class, are obtained.

- The topological transversality theorem for general multivalued maps which have selections in a given class of maps is studied in [11].

- The authors in [12], formulate a new differential operator with complex coefficients in the open unit disk and generalize a class of Briot-Bouquet differential equations. Some inequalities involving the subordination concept were investigated.

- In [13], the solvability of a nonlocal inverse boundary value problem for a mixed pseudohyperbolic-pseudoelliptic integro-differential equation with spectral parameters is discussed. The unique solvability of the inverse boundary value problem for regular values of spectral parameters is proved. For irregular values of spectral parameters, a criterion of existence of an infinite set of solutions of the inverse boundary value problem is established.

- A complete Lyapunov-type characterization of exponential stability for linear skew-product three-parameter semiflows with discrete time is obtained in [14]. More precisely, the authors proved that exponential stability can be described in terms of the existence of appropriate quadratic Lyapunov functions. By applying these results, they prove that the notion of an exponential stability persists under sufficiently small linear perturbations.

- In [15], the existence of solutions for a new class of boundary value problems for fractional differential equations and inclusions, involving right-Caputo and left-Riemann-Liouville fractional derivatives of different orders and right-left Riemann-Liouville fractional integrals with nonlocal boundary conditions is discussed. The existence of solutions for the single-valued case relies on Sadovskii's fixed point theorem, while the existence results for the multi-valued case are proved by applying Bohnenblust-Karlin's and Martelli's fixed point theorems.

- The authors of [16], introduce and study a new derivative called generalized nabla derivative for fuzzy functions on time scales via Hukuhara difference and studies some basic properties. In addition, they prove a fundamental theorem of nabla integral calculus for fuzzy functions on time scales under generalized differentiability on time scales.

- In [17], the existence and uniqueness of solutions for a class of multi-point and integral boundary value problems of multi-term fractional differential equations are discussed. Existence results are proved by means of Krasnosel'skii fixed point theorem and Leray-Schauder nonlinear alternative, while Banach contraction mapping principle is applied to establish the uniqueness of solutions for the given problem.

- In [18], an iterative projection method for solving linear and nonlinear hypersingular integral equations has been proposed. The method is based on the use of sufficient conditions for asymptotic stability of ordinary differential equations systems. The advantage of the method for linear equations is in simplicity of unique solvability verification for the approximate equations system in terms of 
the operator logarithmic norm. The advantage of the method for nonlinear equations is that neither the existence or reversibility of the nonlinear operator derivative is required.

- The periodic behavior of solutions of nonlinear difference equations is studied in [19], where the authors use a new method to find the necessary and sufficient conditions for the existence of periodic solutions.

- In [20], the authors discuss several techniques for starting with a given global solution to an original Multiplicatively Advanced Differential Equations (MADEs) and then generating solutions of new related MADEs, which involve only simple exponentials and trigonometric functions.

- In [21], an asymptotic solution of the linear Cauchy problem in the presence of a "weak" turning point for the limit operator is constructed by the method of $\mathrm{S}$. A. Lomov regularization. The main singularities of this problem are written out explicitly. The asymptotic convergence of a regularized series is also proven.

- The authors of [22], analyze the modified Helmholtz equation in the regular hexagon with symmetric Dirichlet boundary conditions, using the unified transform, also known as the Fokas method. The main achievement of this work concerns the fact that their analysis yields the solution for the case of odd symmetric Dirichlet data in the closed form.

- In [23], the authors apply the Horn-Karlsson approach to find convergence regions for triple $q$-hypergeometric functions.

Funding: This research received no external funding.

Conflicts of Interest: The authors declare no conflict of interest.

\section{References}

1. Kucukoglu, I.; Simsek, B.; Simsek, Y. Generating Functions for New Families of Combinatorial Numbers and Polynomials: Approach to Poisson-Charlier Polynomials and Probability Distribution Function. Axioms 2019, 8, 112. [CrossRef]

2. Aleroev, T. On One Problems of Spectral Theory for Ordinary Differential Equations of Fractional Order. Axioms 2019, 8, 117. [CrossRef]

3. Shah, R.; Khan, H.; Baleanu, D. Fractional Whitham-Broer-Kaup Equations within Modified Analytical Approaches. Axioms 2019, 8, 125. [CrossRef]

4. Ricci, P.E.; Natalini, P. General Linear Recurrence Sequences and Their Convolution Formulas. Axioms 2019, 8, 132. [CrossRef]

5. Li, C.H.; Plowman, H. Solutions of the Generalized Abel's Integral Equationsof the Second Kind with Variable Coefficients. Axioms 2019, 8, 137. [CrossRef]

6. Cho, N.E.; Dziok, J. Harmonic Starlike Functions with Respect to Symmetric Points. Axioms 2020, 9, 3. [CrossRef]

7. Park, C.H.; Moaaz O.; Bazighifan, O. Oscillation Results for Higher Order Differential Equations. Axioms 2020, 9, 14. [CrossRef]

8. Shaikhet, L. Stability of Equilibria of Rumor Spreading Model under Stochastic Perturbations. Axioms 2020, 9, 24. [CrossRef]

9. Shah, N.H.; Sheoran N.; Shah, Y. Dynamics of HIV-TB Co-Infection Model. Axioms 2020, 9, 29. [CrossRef]

10. Yousef, A.T.; Salleh, Z. On a Harmonic Univalent Subclass of Functions Involving a Generalized Linear Operator. Axioms 2020, 9, 32. [CrossRef]

11. O'Regan, D. Coincidence Continuation Theory for Multivalued Maps with Selections in a Given Class. Axioms 2020, 9, 37. [CrossRef]

12. Ibrahim, R.W.; Elobaid, R.M.; Obaiys, S.J. Generalized Briot-Bouquet Differential Equation Based on New Differential Operator with Complex Connections. Axioms 2020, 9, 42. [CrossRef]

13. Yuldashev, T.K. Nonlocal Inverse Problem for a Pseudohyperbolic- PseudoellipticType Integro-Differential Equations. Axioms 2020, 9, 45. [CrossRef]

14. Dragicěvic, D.; Preda, C. Lyapunov Type Theorems for Exponential Stability of Linear Skew-Product Three-Parameter Semiflows with Discrete Time. Axioms 2020, 9, 47. [CrossRef] 
15. Alsaedi, A.; Broom, A.; Ntouyas S.K.; Ahmad, A. Nonlocal Fractional Boundary Value Problems Involving Mixed Right and Left Fractional Derivatives and Integrals. Axioms 2020, 9, 50. [CrossRef]

16. Leelavathi, R.; Kumar, G.S.; Agarwal, R.P.; Wang, C.H.; Murty, M.S.N. Generalized Nabla Differentiability and Integrability for Fuzzy Functions on Time Scales. Axioms 2020, 9, 65. [CrossRef]

17. Ahmad, B.; Alghamdi, N.; Alsaedi A.; Ntouyas, S.K. Existence Results for Nonlocal Multi-Point and Multi-Term Fractional Order Boundary Value Problems. Axioms 2020, 9, 70. [CrossRef]

18. Boykov, I.; Roudnev, V.; Boykova, A. Approximate Methods for Solving Linear and Nonlinear Hypersingular Integral Equations. Axioms 2020, 9, 74. [CrossRef]

19. Moaaz, O.; Mahjoub, H.; Muhib, A. On the Periodicity of General Class of Difference Equations. Axioms 2020, 9, 75. [CrossRef]

20. Moaaz, O.; Mahjoub, H.; Muhib, A. Eigenfunction Families and Solution Bounds for Multiplicatively Advanced Differential Equations. Axioms 2020, 9, 83.

21. Yeliseev, A. On the Regularized Asymptotics of a Solution to the Cauchy Problem in the Presence of a Weak Turning Point of the Limit Operator. Axioms 2020, 9, 86. [CrossRef]

22. Kalimeris K.; Fokas, A.S. The Modified Helmholtz Equation on a Regular Hexagon-The Symmetric Dirichlet Problem. Axioms 2020, 9, 89. [CrossRef]

23. Ernst, T. On the Triple Lauricella-Horn-Karlsson $q$-Hypergeometric Functions. Axioms 2020, 9, 93. [CrossRef]

Publisher's Note: MDPI stays neutral with regard to jurisdictional claims in published maps and institutional affiliations.

(C) 2020 by the authors. Licensee MDPI, Basel, Switzerland. This article is an open access article distributed under the terms and conditions of the Creative Commons Attribution (CC BY) license (http://creativecommons.org/licenses/by/4.0/). 